\title{
Manipulating Assemblies In Metallosupramolecular Gels, Driven by Isomeric Ligands, Metal Coordination And Adaptive Binary Gelator Systems
}

Arnab Dawn ${ }^{a *}$, Jinnipha Pajoubpong ${ }^{a}$, Amira Mesmer ${ }^{a}$, Marzieh Mirzamani a, Lilin $\mathrm{He}^{\mathrm{b}}$, and Harshita

Kumari ${ }^{a *}$

a James L. Winkle College of Pharmacy, University of Cincinnati, 231 Albert Sabin Way, Medical Science

Building, Cincinnati, OH, 45267-0514, USA.

${ }^{b}$ Neutron Scattering Division, Oak Ridge National Laboratory, Oak Ridge, TN 37831, USA.

*Email: arnabdawn16@gmail.com

*Email: kumariha@ucmail.uc.edu 

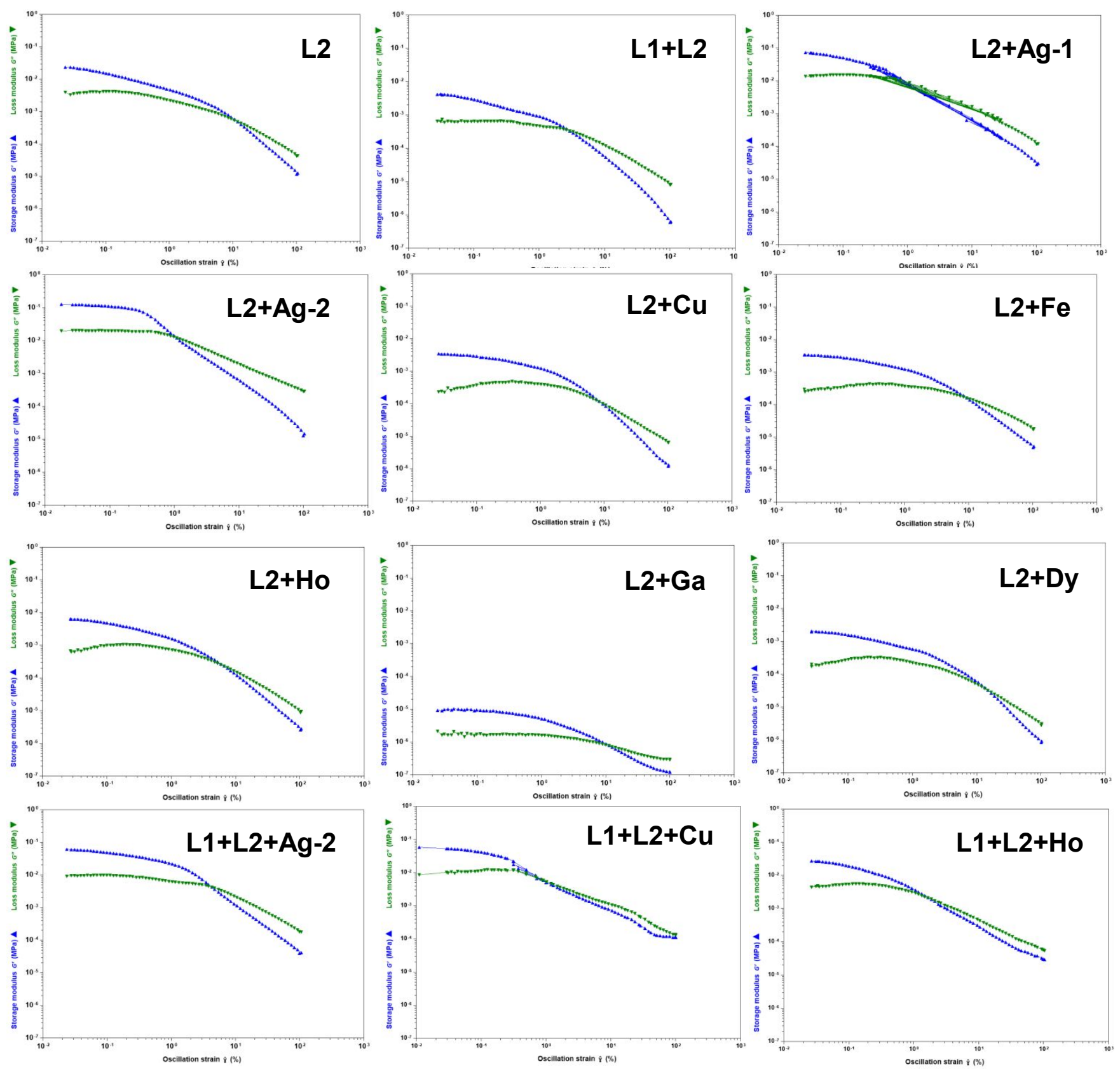

Figure S1. Strain sweep experiments performed at a constant frequency of $1 \mathrm{~Hz}$ on gel samples prepared at total ligand concentration $2 \%(\mathrm{w} / \mathrm{v})$ (storage and loss moduli are represented by blue and green triangles, respectively). 

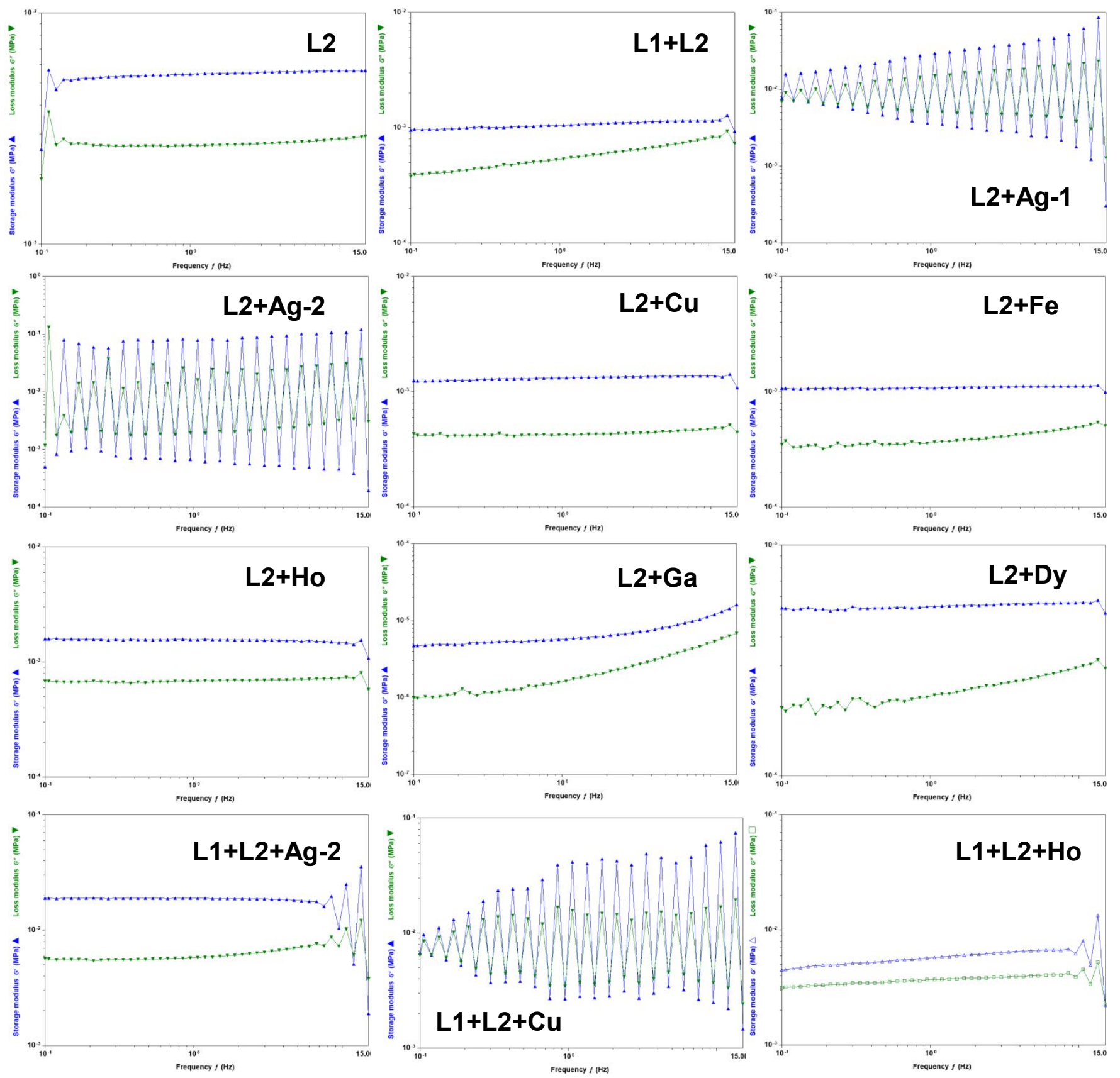

Figure S2. Frequency sweep experiments performed at a constant strain of $1 \%$ on gel samples prepared at total ligand concentration $2 \%(\mathrm{w} / \mathrm{v})$ (storage and loss moduli are represented by blue and green triangles, respectively). The inconsistent data points in case of $\mathbf{L} \mathbf{2}+\mathbf{A g}-\mathbf{1}, \mathbf{L} \mathbf{2}+\mathbf{A g}-\mathbf{2}$ and $\mathbf{L 1}+\mathbf{L} \mathbf{2}+\mathbf{C u}$ are because of sample texture and not related to frequency dependency. 


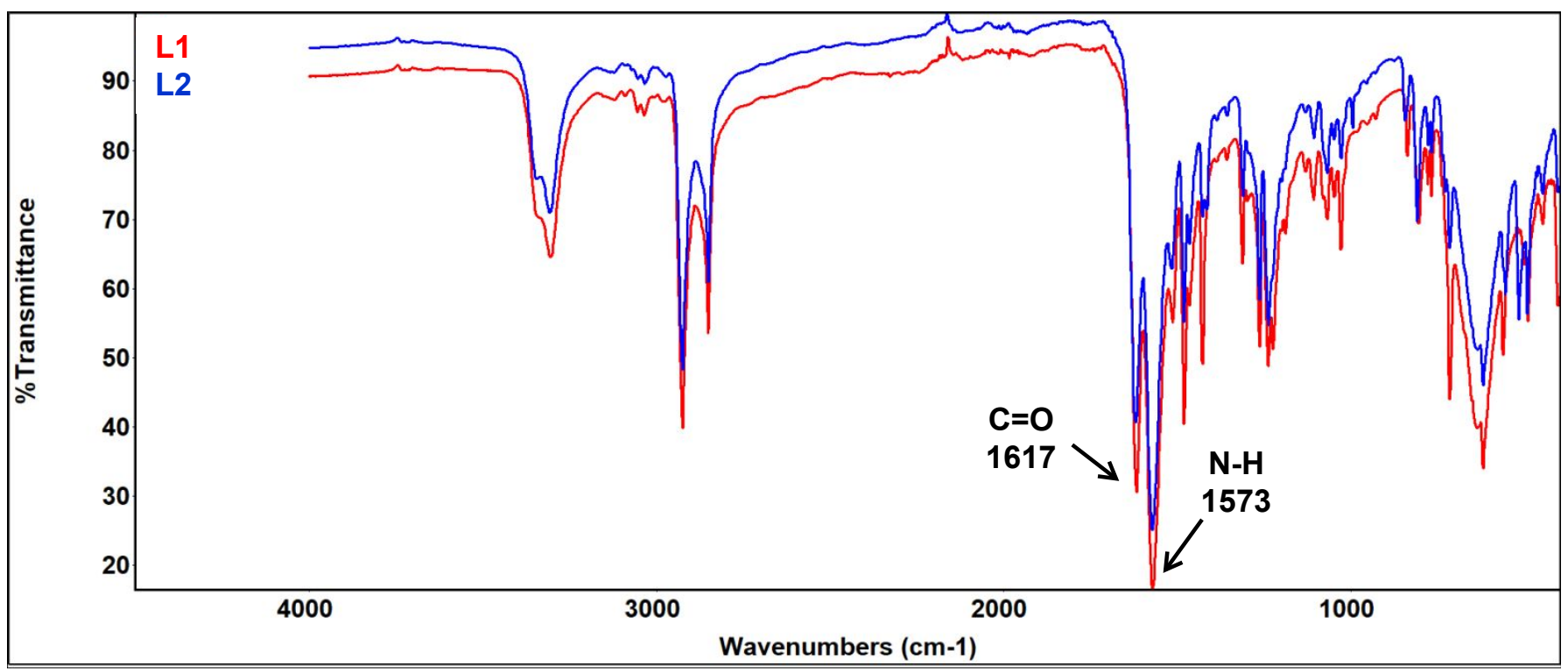

Figure S3. FT-IR spectra of synthesized L1 and L2 solids. 


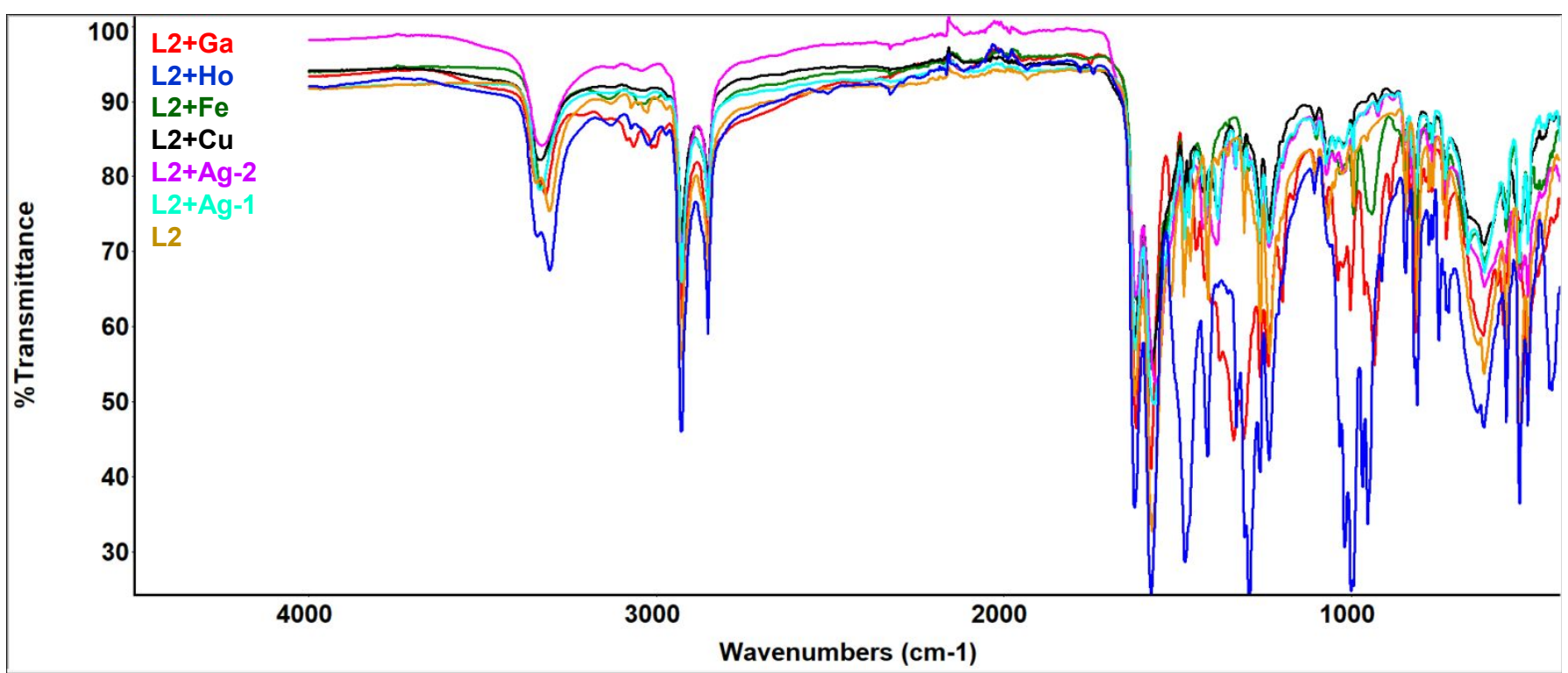

Figure S4. FT-IR spectra of various xerogels of $\mathbf{L} 2$ in presence and in absence of metal ions.

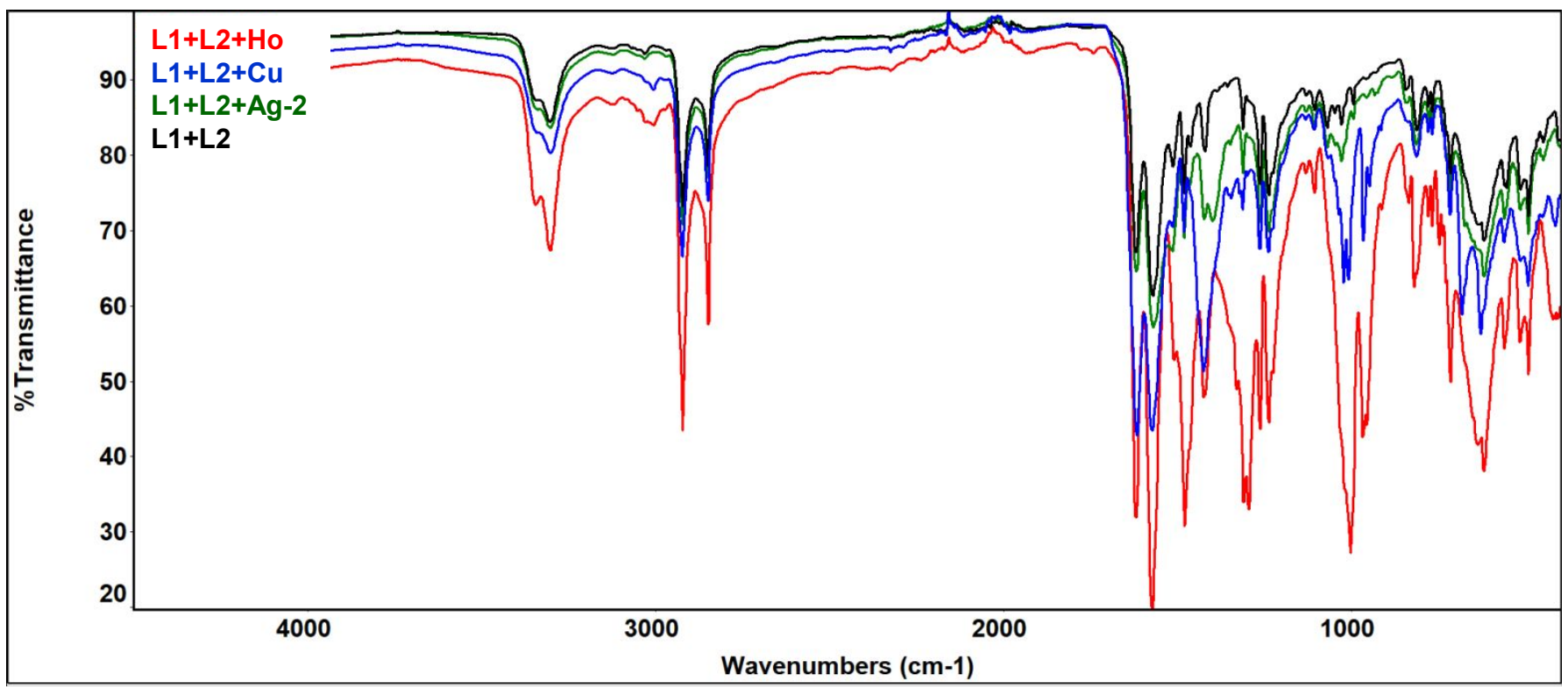

Figure S5. FT-IR spectra of various xerogels of $\mathbf{L 1}+\mathbf{L} 2$ in presence and in absence of metal ions. 


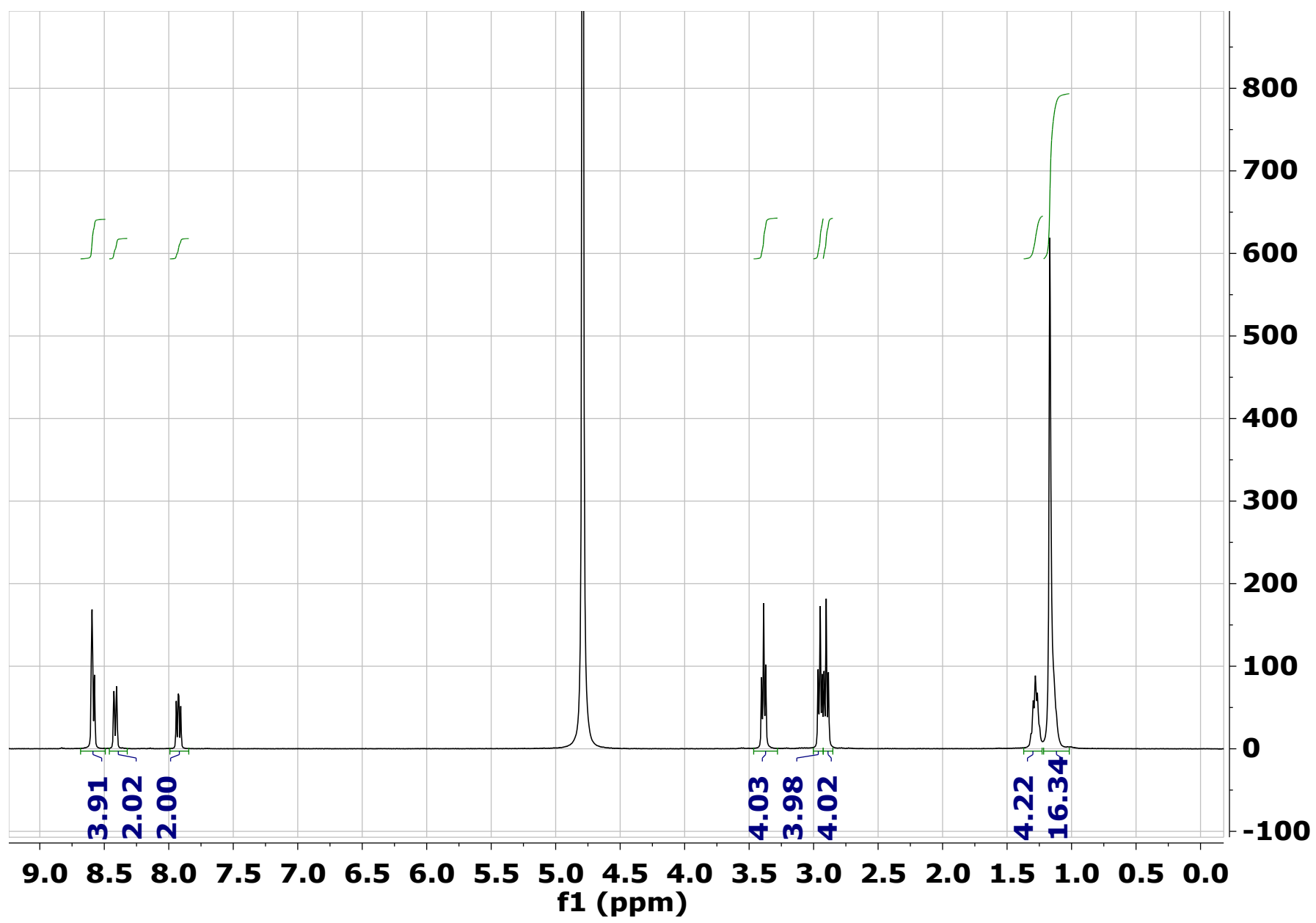

Figure S6. ${ }^{1} \mathrm{H} \mathrm{NMR}$ spectra of $\mathbf{L 1}$ in $\mathrm{D}_{2} \mathrm{O} / \mathrm{HCl}\left(10 \mu \mathrm{L} 12 \mathrm{~N} \mathrm{HCl}\right.$ added to $\left.1 \mathrm{~mL} \mathrm{D}_{2} \mathrm{O}\right)$. The spectrum was calibrated against residual water peak at $4.79 \mathrm{ppm}$. 


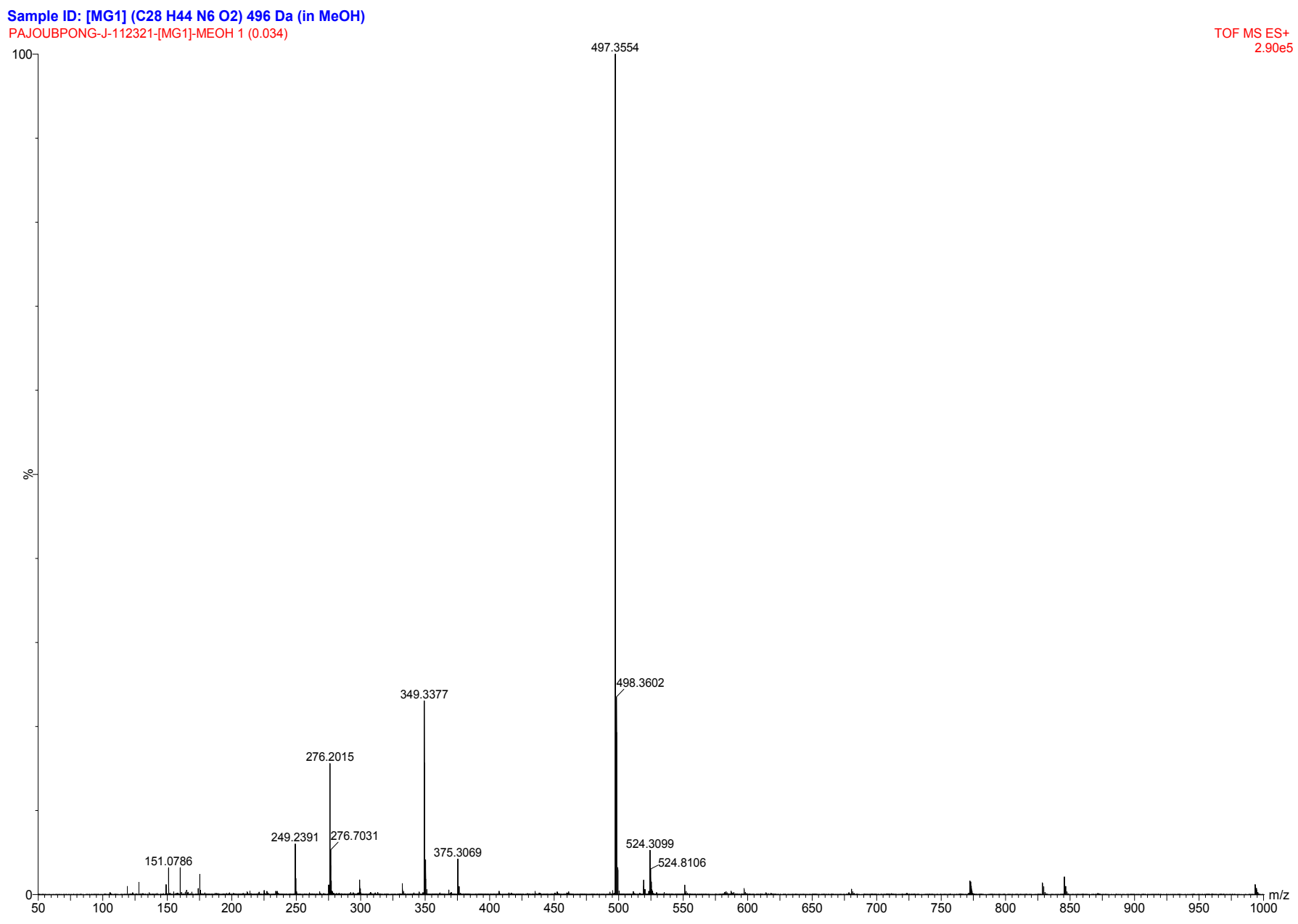

Figure S7. ESI Mass spectra of L1 




Figure S8. ${ }^{1} \mathrm{H}$ NMR spectra of $\mathbf{L} 2$ in DMSO- $d_{6}$. 


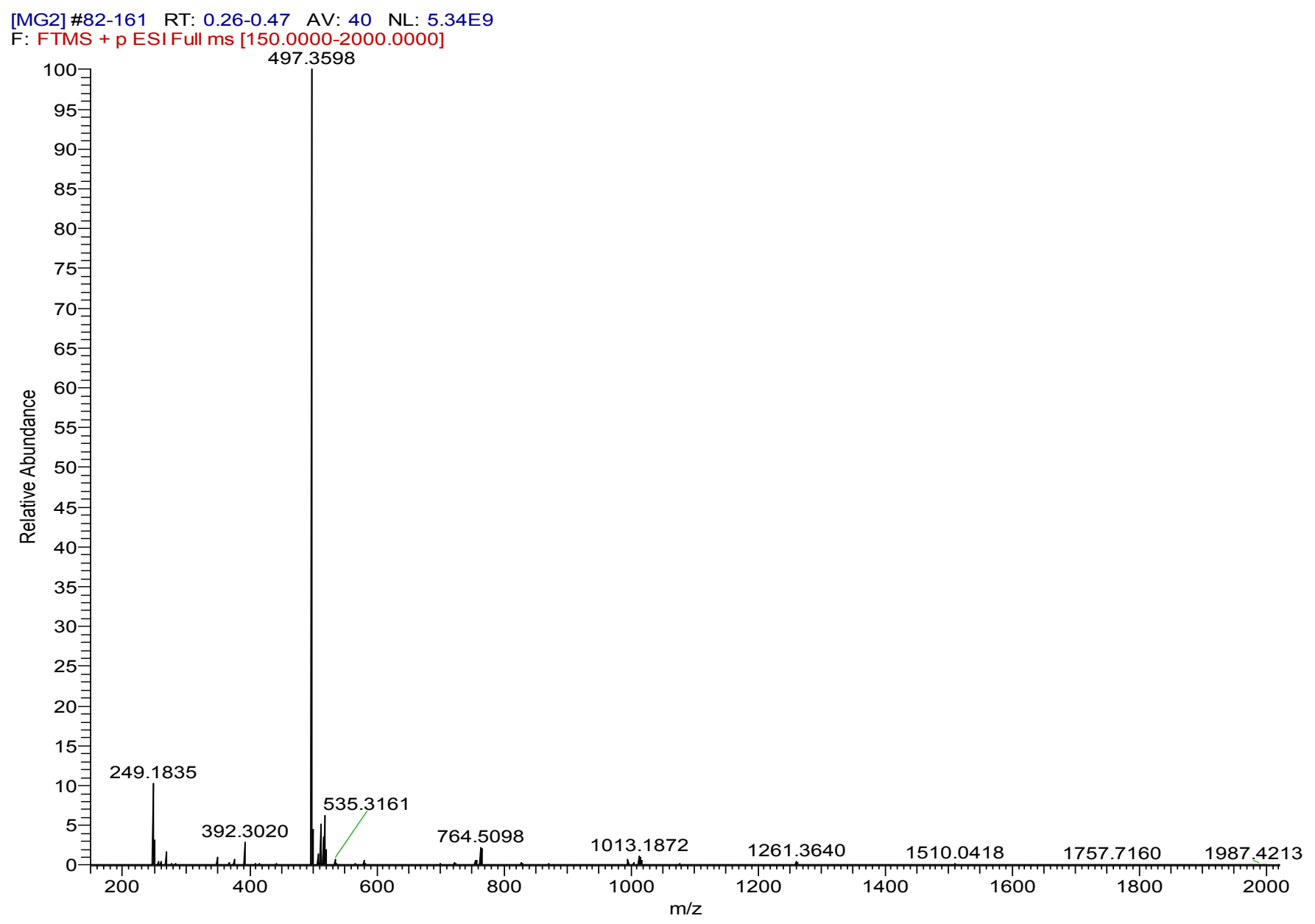

Figure S9. ESI Mass spectra of L2 
Table S1. SANs fitting parameters for gel $\mathbf{L 2}, \mathbf{L 2}+\mathbf{C u}$ used in an unsmeared combined Power Law and Gaussian peak models. The fits were performed by using Igor Pro.

\begin{tabular}{|l|l|l|}
\hline & L2 & $\mathbf{L 2}+\mathbf{C u}$ \\
\hline Coefficient, A & $2.45 \times 10^{-7} \pm 3.74 \times 10^{-10}$ & $3.37 \times 10^{-7} \pm 4.37 \times 10^{-10}$ \\
\hline$(-)$ Power & $4 \pm 0.00037$ & $3.98 \pm 0.00031$ \\
\hline Scale Factor, $\mathrm{I}_{0}$ & $0.0105 \pm 0.00042$ & $0.00646 \pm 0.00043$ \\
\hline Peak position $\left(\AA^{-1}\right)$ & $0.2904 \pm 0.00097$ & $0.28509 \pm 0.00151$ \\
\hline Std Dev $\left(\AA^{-1}\right)$ & $0.0172 \pm 0.00081$ & $0.01621 \pm 0.00122$ \\
\hline Incoherent Bgd $\left(\mathrm{cm}^{-1}\right)$ & $0.0492 \pm 0.00012$ & $0.05329 \pm 0.00012$ \\
\hline Sqrt $\left(\square^{2} / \mathrm{N}\right)$ & 3.04819 & 1.3834 \\
\hline
\end{tabular}
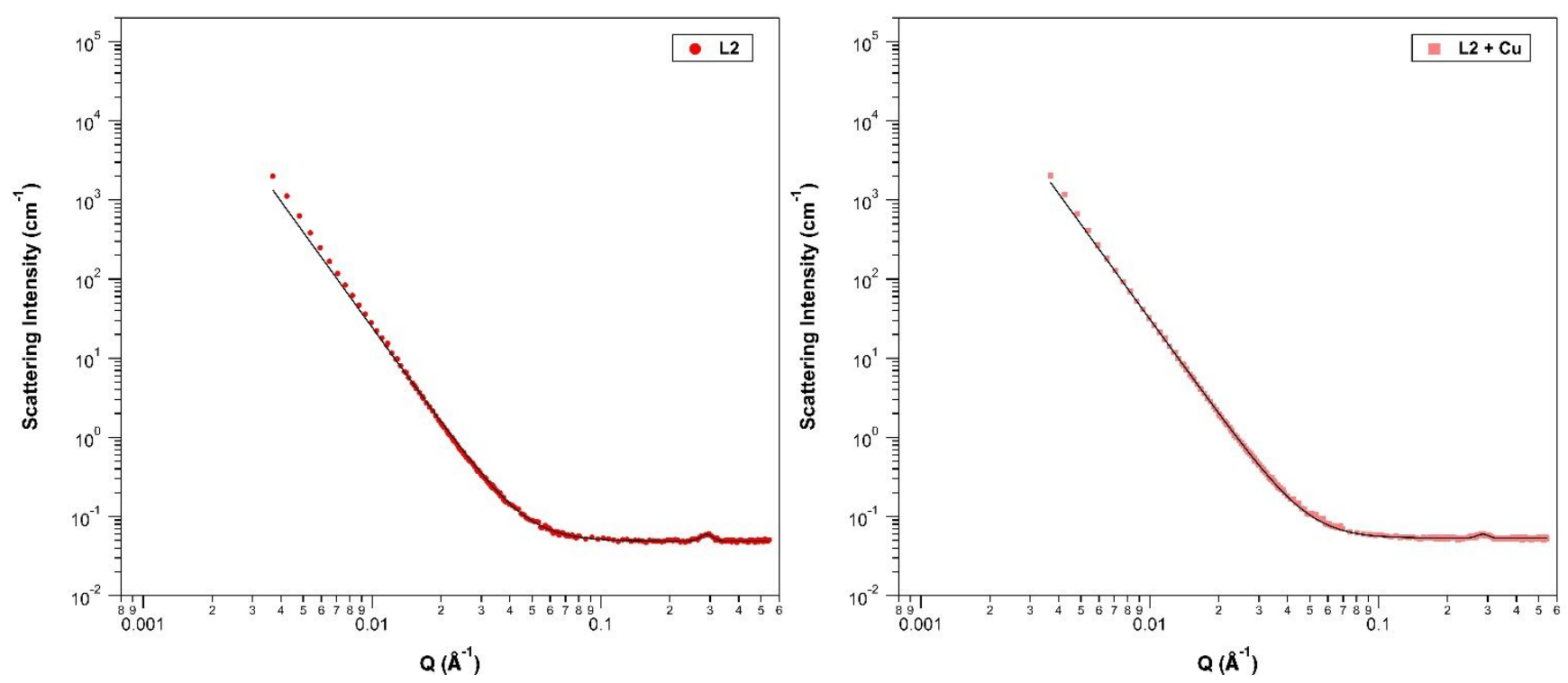

Figure S10. Fitted SANS profiles for $\mathbf{L 2}$ and $\mathbf{L 2}+\mathbf{C u}$. The solid lines represent the model fits to the data using the fitting parameters in Table S1. 
Table S2. SANs fitting parameters for gel $\mathbf{L 2}+\mathbf{A g}-\mathbf{2}, \mathbf{L 2}+\mathbf{A g}-\mathbf{1}, \mathbf{L 2}+\mathbf{G a}, \mathbf{L 2}+\mathbf{D y}$, and $\mathbf{L 2}+\mathbf{F e}$ used in an unsmeared combined Guinier-Porod and Gaussian peak models. The fits were performed by using Igor Pro.

\begin{tabular}{|l|l|l|l|l|l|}
\hline & L2+Ag-2 & L2+Ag-1 & L2+Ga & L2+Dy & L2+Fe \\
\hline Guinier scale & $0.00828 \pm 4.47 \times 10^{-6}$ & $0.01024 \pm 4.12 \times 10^{-7}$ & $0.00025 \pm 1.51 \times 10^{-8}$ & $0.00561 \pm 2.59 \times 10^{-7}$ & $0.00670 \pm 3.26 \times 10^{-7}$ \\
\hline Dimension variable, $\mathrm{s}$ & $2.02 \pm 0.00010$ & $2.27 \pm 9.45 \times 10^{-6}$ & $2.69 \pm 1.54 \times 10^{-5}$ & $2.40 \pm 9.64 \times 10^{-6}$ & $2.22 \pm 1.29 \times 10^{-5}$ \\
\hline $\operatorname{Rg}(\AA)$ & $103.675 \pm 0.14589$ & $86.431 \pm 0.00278$ & $27.200 \pm 0.00184$ & $105.525 \pm 0.00391$ & $69.431 \pm 0.00210$ \\
\hline Porod Exponent & $2.40 \pm 0.00029$ & $3.63 \pm 4.98 \times 10^{-5}$ & $4 \pm 0.00025$ & $4 \pm 6.35 \times 10^{-5}$ & $4 \pm 0.00011$ \\
\hline Scale Factor, $\mathrm{I}_{0}$ & $0.0166 \pm 0.00123$ & $0.0392 \pm 4.34 \times 10^{-5}$ & $0.0061 \pm 1.55 \times 10^{-5}$ & $0.0104 \pm 2.10 \times 10^{-5}$ & $0.0098 \pm 1.86 \times 10^{-5}$ \\
\hline Peak position $\left(\AA^{-1}\right)$ & $0.1585 \pm 0.00289$ & $0.1464 \pm 2.47 \times 10^{-5}$ & $0.2909 \pm 0.00012$ & $0.2832 \pm 6.34 \times 10^{-5}$ & $0.2827 \pm 8.06 \times 10^{-5}$ \\
\hline Std Dev $\left(\AA^{-1}\right)$ & $0.0048 \pm 0.00080$ & $0.0162 \pm 1.95 \times 10^{-5}$ & $0.0384 \pm 0.00010$ & $0.0266 \pm 5.62 \times 10^{-5}$ & $0.0317 \pm 6.71 \times 10^{-5}$ \\
\hline Incoherent Bgd $\left(\mathrm{cm}^{-1}\right)$ & $0.0460 \pm 0.00013$ & $0.0774 \pm 6.48 \times 10^{-6}$ & $0.0622 \pm 5.10 \times 10^{-6}$ & $0.0688 \pm 5.55 \times 10^{-6}$ & $0.0566 \pm 5.68 \times 10^{-6}$ \\
\hline Sqrt $\left(\square^{2} / \mathrm{N}\right)$ & 6.0155 & $63.6957^{*}$ & $86.9127^{*}$ & $122.222^{*}$ & $82.3684^{*}$ \\
\hline
\end{tabular}

Table S3. SANs fitting parameters for gel $\mathbf{L 2}+\mathbf{H o}, \mathbf{L 1}+\mathbf{L} 2, \mathbf{L 1}+\mathbf{L} 2+\mathbf{H o}, \mathbf{L 1}+\mathbf{L} \mathbf{2}+\mathbf{C u}$, and $\mathbf{L 1}+\mathbf{L} \mathbf{2}+\mathbf{A g}-\mathbf{2}$ used in an unsmeared combined Guinier-Porod, DAB, and Gaussian peak models. The fits were performed by using SasView.

\begin{tabular}{|l|l|l|l|l|l|}
\hline & L2+Ho & L1+L2 & L1+L2+Ho & L1+L2+Cu & L1+L2+Ag-2 \\
\hline Guinier scale & $0.00078 \pm 1.19 \times 10^{-6}$ & $0.00021 \pm 1.99 \times 10^{-7}$ & $0.00157 \pm 7.02 \times 10^{-8}$ & $0.00217 \pm 1.22 \times 10^{-7}$ & $0.00142 \pm 1.35 \times 10^{-6}$ \\
\hline Dimension variable, $\mathrm{s}$ & $2.82 \pm 0.00064$ & $2.95 \pm 0.00016$ & $2.52 \pm 1.22 \times 10^{-5}$ & $2.53 \pm 1.31 \times 10^{-5}$ & $2.53 \pm 0.00027$ \\
\hline Rg $(\AA)$ & $89.259 \pm 0.11446$ & $14.899 \pm 0.01418$ & $43.600 \pm 0.00171$ & $84.429 \pm 0.00379$ & $61.064 \pm 0.04939$ \\
\hline Porod Exponent & $4.0 \pm 6.04 \times 10^{-9}$ & $3.98 \pm 0.00127$ & $3.83 \pm 1.09 \times 10^{-4}$ & $4.0 \pm 6.40 \times 10^{-9}$ & $3.71 \pm 0.00105$ \\
\hline DAB scale & $0.00043 \pm 1.55 \times 10^{-6}$ & $0.00054 \pm 4.22 \times 10^{-6}$ & $0.00042 \pm 1.03 \times 10^{-7}$ & $0.00061 \pm 1.90 \times 10^{-7}$ & $0.00032 \pm 1.29 \times 10^{-6}$ \\
\hline Correlation length $(\AA)$ & $7.074 \pm 0.01310$ & $4.757 \pm 0.01652$ & $7.411 \pm 9.64 \times 10^{-4}$ & $5.230 \pm 0.00075$ & $8.882 \pm 0.01831$ \\
\hline Scale Factor, $I_{0}$ & $0.0255 \pm 3.21 \times 10^{-4}$ & $0.0105 \pm 5.61 \times 10^{-4}$ & $0.0081 \pm 3.62 \times 10^{-5}$ & $0.0184 \pm 4.45 \times 10^{-5}$ & $0.0139 \pm 0.00039$ \\
\hline Peak position $\left(\AA^{-1}\right)$ & $0.2892 \pm 3.02 \times 10^{-4}$ & $0.2150 \pm 0.00113$ & $0.2094 \pm 7.36 \times 10^{-5}$ & $0.1876 \pm 3.27 \times 10^{-5}$ & $0.1999 \pm 0.00134$ \\
\hline Std Dev $\left(\AA^{-1}\right)$ & $0.0182 \pm 2.47 \times 10^{-4}$ & $0.0143 \pm 9.00 \times 10^{-4}$ & $0.0122 \pm 6.09 \times 10^{-5}$ & $0.0101 \pm 2.71 \times 10^{-5}$ & $0.0405 \pm 0.00120$ \\
\hline Incoherent Bgd $\left(\mathrm{cm}^{-1}\right)$ & $0.0780 \pm 8.93 \times 10^{-5}$ & $0.0593 \pm 1.28 \times 10^{-4}$ & $0.0645 \pm 5.81 \times 10^{-6}$ & $0.0743 \pm 5.94 \times 10^{-6}$ & $0.0631 \pm 1.30 \times 10^{-4}$ \\
\hline Sqrt $(\square / \mathrm{N})$ & 10.545 & 3.1753 & $1729.1^{2}$ & $5158.2 *$ & 1.9744 \\
\hline
\end{tabular}

*The reduced chi-squared reported is unrealistic chi-squared values. This is likely the cause of the error estimates on the low-q intensity, which consistently reported from the model fits to the data consisting of the low-q region $(0.001<\mathrm{Q}<0.003)$, although the models visually fit well. Therefore, these large, reduced chi-squared does not truly represent the statistical uncertainties. 

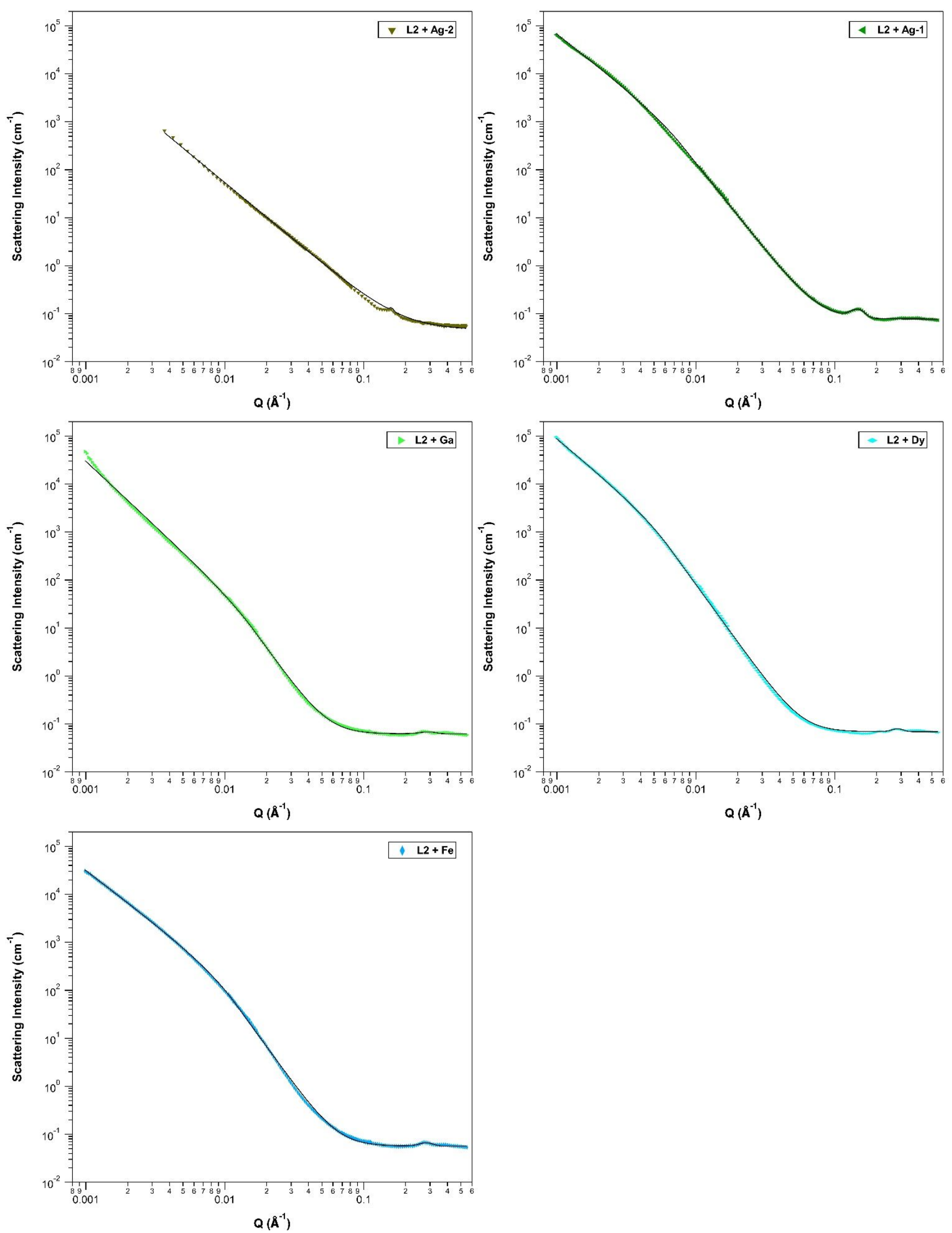

Figure S11. Fitted SANS profiles for L2+Ag-2, L2+Ag-1, L2+Ga, L2+Dy, and L2+Fe. The solid lines represent the model fits to the data using the fitting parameters in Table S2. 

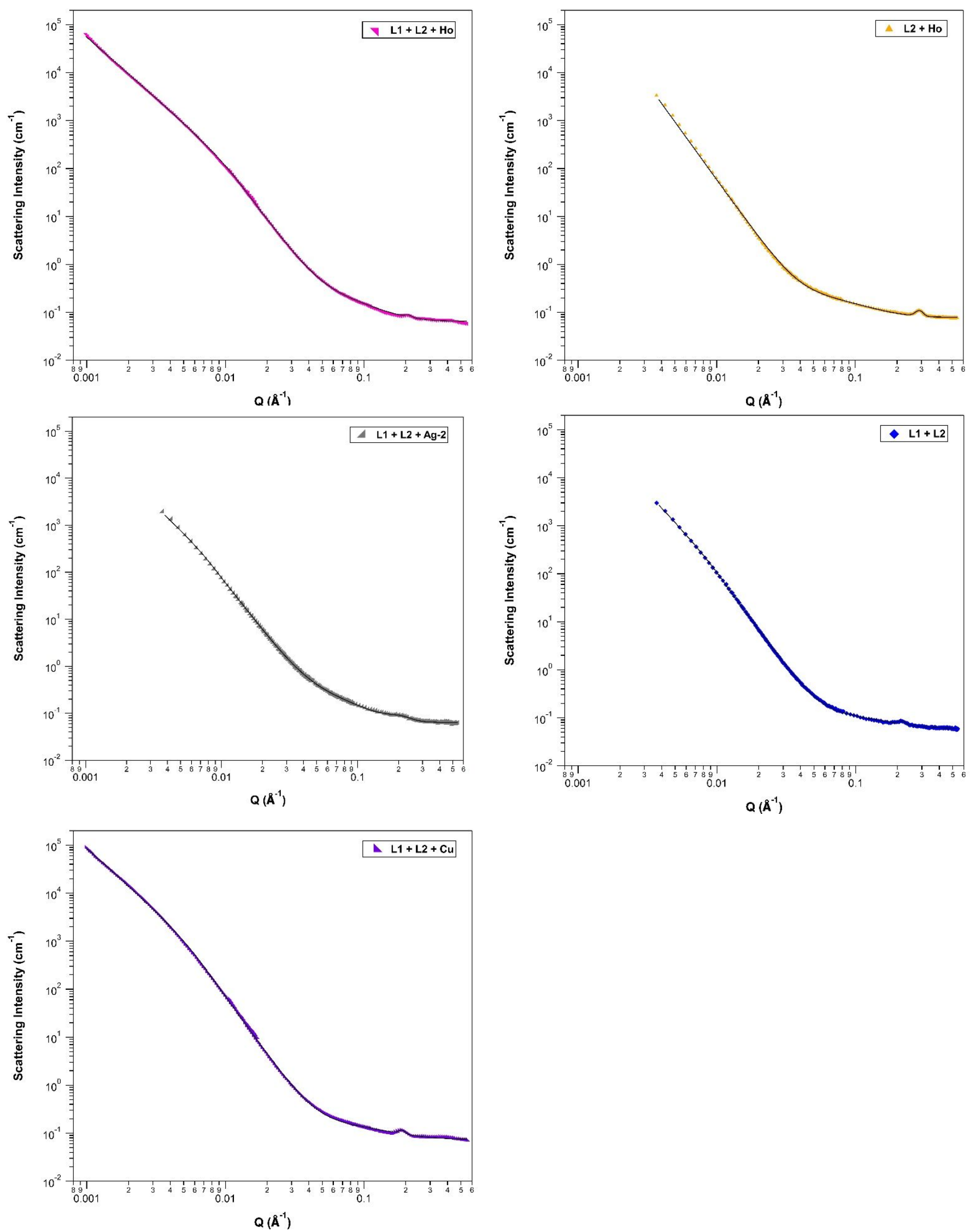

Figure S12. Fitted SANS profiles for $\mathbf{L} 2+\mathbf{H o}, \mathbf{L 1}+\mathbf{L} 2, \mathbf{L 1}+\mathbf{L} 2+\mathbf{H o}, \mathbf{L} 1+\mathbf{L} 2+\mathbf{C u}$, and $\mathbf{L 1}+\mathbf{L} 2+\mathbf{A g}-\mathbf{2}$. The solid lines represent the model fits to the data using the fitting parameters in Table S3. 